\title{
On values of a modular form on $\Gamma_{0}(N)$
}

\section{Chol (Pohang)}

1. Introduction. The values of a modular function at certain points play a role in modular form theory. Especially, those of $j(z)$ are related to other number theoretical objects, where $j(z)$ is the usual elliptic modular function on $\mathrm{SL}_{2}(\mathbb{Z})$. For example, if a complex number $\tau$ is a Heegner point, i.e. $\tau=\left(-b+\sqrt{b^{2}-4 a c}\right) / 2 a$ with $a, b, c \in \mathbb{Z}, \operatorname{gcd}(a, b, c)=1$ and $b^{2}-4 a c<0$, then the Hurwitz-Kronecker class number is related to the trace of $j(\tau)$ that is called a singular modulus in [9].

After Borcherds' work [2] on the infinite product expansion of modular forms with no divisor except cusps and Heegner points, some results give connections between the values of a modular function at divisor points and the exponents of the infinite product expansion of modular forms. Bruinier, Kohnen and Ono provided a relation between the infinite product expansion of a modular form $f$ and the values of a certain meromorphic modular form at points in the divisor of $f$ (see [3]). Ahlgren gave analogues of these results for modular forms on $\Gamma_{0}(p)$ for $p \in\{2,3,5,7,13\}$ (see [1]). Their results are restricted to the genus zero group.

In this paper, we give analogues of their results for $\Gamma_{0}(N)$ for square free $N$, and also describe the values of a modular function at certain points. To do this we consider a certain sequence of modular functions. In Section 2, we state the connection between the exponents of an infinite product expansion of a modular form $f$ and the values of a modular function at divisor points of $f$, where $\theta f=\frac{1}{2 \pi i} f^{\prime}(z)$. In Section 3, we define $(l, N)$-type sequences of modular functions and give some related identities. In Section 4, we obtain congruence properties of values of a modular function.

2. The values of a modular function at divisor points. Suppose that $N$ is a square free positive integer. The group $\Gamma_{0}(N)$ is the congruence

2000 Mathematics Subject Classification: Primary 11F11; Secondary 11F33.

Key words and phrases: modular forms, one variable, congruences for modular forms.

This work is partially supported by KOSEF R01-2003-00011596-0. 
subgroup of $\mathrm{SL}_{2}(\mathbb{Z})$ defined as

$$
\Gamma_{0}(N)=\left\{\left(\begin{array}{ll}
a & b \\
c & d
\end{array}\right) \in \mathrm{SL}_{2}(\mathbb{Z}) \mid c \equiv 0(\bmod N)\right\} .
$$

Let $\Gamma$ denote $\mathrm{SL}_{2}(\mathbb{Z})$ and $\mathcal{F}_{N}$ be a fundamental domain for the action of $\Gamma_{0}(N)$ on $\mathbb{H}$. We denote the set of distinct cusps by $S_{N}$,

$$
S_{N}=\{1 / w \mid w \neq N \text { and } w \mid N\} \cup\{0, \infty\} .
$$

From now on, we suppose that if $t$ is a cusp point, then $t$ is in $S_{N}$. The period of $q$-expansion at $t$ is denoted by $N_{t}$, where $N_{t}$ is given by the following way:

$$
N_{t}= \begin{cases}N t & \text { if } t \in S_{N} \backslash\{0, \infty\} \\ 1 & \text { if } t=\infty \\ N & \text { if } t=0\end{cases}
$$

Adjoining the cusps to $\Gamma_{0}(N) \backslash \mathbb{H}$, we obtain a compact Riemann surface $X_{0}(N)$. For $\tau \in \mathbb{H} \cup S_{N}$, let $Q_{\tau}$ be the image of $\tau$ under the canonical map from $\mathbb{H} \cup S_{N}$ to $X_{0}(N)$.

Suppose $G$ is a meromorphic modular form of weight 2 on $\Gamma_{0}(N)$. The residue of $G$ at $Q_{\tau}$ on $X_{0}(N)$, denoted by $\operatorname{Res}_{Q_{\tau}} G d z$, is well defined since we have the canonical correspondence between meromorphic modular forms of weight 2 on $\Gamma_{0}(N)$ and meromorphic 1 -forms of $X_{0}(N)$. If $\operatorname{Res}_{\tau} G$ denotes the residue of $G$ at $\tau$ on $\mathbb{H}$, then for $\tau \in \mathbb{H}$ we obtain

$$
\operatorname{Res}_{Q_{\tau}} G d z=\frac{1}{l_{\tau}} \operatorname{Res}_{\tau} G .
$$

Here, $l_{\tau}$ is the order of the isotropy group at $\tau$. In particular, if $f$ is a meromorphic modular form of weight $k$ on $\Gamma_{0}(N)$ and $G=\frac{\theta f}{f}$, then the residue of $G$ at $Q_{\tau}$ on $\tau \in \mathbb{H}$ is computed from the order of zero or pole of $f$ at $\tau \in \mathbb{H}$. The latter is denoted by $\nu_{\tau}^{(N)}(f)$ and has the form

$$
\nu_{\tau}^{(N)}(f)=\frac{1}{l_{\tau}} \operatorname{ord}_{\tau}(f),
$$

where $\operatorname{ord}_{\tau}(f)$ denotes the order of zero or pole of $f$ at $\tau$ as a complex function on $\mathbb{H}$. Then we have

$$
2 \pi i \cdot \operatorname{Res}_{Q_{\tau}} \frac{\theta f}{f}=\nu_{\tau}^{(N)}(f)
$$

We introduce some notations to describe $\operatorname{Res}_{Q t} G d z$ at every cusp $t$. First, recall the usual slash operator $\left.f(z)\right|_{k} \gamma$ given as

$$
\left.f(z)\right|_{k} \gamma=\operatorname{det}(\gamma)^{k / 2}(c z+d)^{-k} f(\gamma z)
$$

where $\gamma=\left(\begin{array}{ll}a & b \\ c & d\end{array}\right) \in \mathrm{GL}_{2}^{+}(\mathbb{Q})$ and $\gamma z$ denotes $\frac{a z+b}{c z+d}$. From now on, $q$ denotes 
$e^{2 \pi i z}$. We define a matrix $\gamma_{t}^{(N)}$ in the following way:

$$
\gamma_{t}^{(N)}:= \begin{cases}\left(\begin{array}{cc}
1 & 0 \\
1 / t & 1
\end{array}\right)\left(\begin{array}{cc}
N_{t} & 0 \\
0 & 1
\end{array}\right) & \text { if } 1 / t \mid N, \\
\left(\begin{array}{cc}
0 & -1 \\
1 & 0
\end{array}\right)\left(\begin{array}{cc}
N & 0 \\
0 & 1
\end{array}\right) & \text { if } t=0, \\
\left(\begin{array}{ll}
1 & 0 \\
0 & 1
\end{array}\right) & \text { if } t=\infty .\end{cases}
$$

If at each cusp, $G$ has the Fourier expansion of the form

$$
\left.G(z)\right|_{2} \gamma_{t}^{(N)}=\sum_{n=m_{t}}^{\infty} a_{t}(n) q^{n} \quad \text { at } \infty
$$

then we have

$$
\operatorname{Res}_{Q_{t}} G d z=\frac{a_{t}(0)}{2 \pi i} \quad \text { for } t \in S_{N}
$$

From now on, we assume that all of the meromorphic modular functions in this paper may have poles only at cusps. Let $g$ be a meromorphic modular function on $\Gamma_{0}(N)$ which at each cusp has the $q$-expansion of the form

$$
\left.g(z)\right|_{0} \gamma_{t}^{(N)}=\sum_{n=\mu_{t}}^{\infty} r_{t}(n) q^{n} \quad \text { at } \infty .
$$

Using these results, we can obtain a connection between the Fourier coefficients of $\frac{\theta f}{f}$ and the values of a meromorphic modular function at the divisor points of $f$, where $f$ is a meromorphic modular form of weight $k$ on $\Gamma_{0}(N)$.

THEOREM 2.1. Suppose that $f(z)$ is a meromorphic modular form of weight $k$ on $\Gamma_{0}(N)$ with square free $N$. For each cusp $t$ let $\left\{c_{t}(n)\right\}_{n=1}^{\infty}$ be the complex numbers for which

$$
\left.f\right|_{k} \gamma_{t}^{(N)}=\alpha_{t} q^{h_{t}} \prod_{n=1}^{\infty}\left(1-q^{n}\right)^{c_{t}(n)} \quad \text { for a complex number } \alpha_{t} .
$$

If $g(z)$ is a meromorphic modular function on $\Gamma_{0}(N)$ with possible poles only at cusps, then

$$
\begin{aligned}
\sum_{t \in S_{N}} \sum_{\mu_{t} \leq n<0}\left(\sum_{d \mid-n} c_{t}(d) d\right) r_{t}(n) & \\
& =\sum_{\tau \in \mathcal{F}_{N}} \nu_{\tau}^{(N)}(f) g(\tau)+\sum_{t \in S_{N}}\left(h_{t}-N_{t} \frac{k}{12}\right) r_{t}(0)+P_{g}
\end{aligned}
$$


where

and $\sigma(n)=\sum_{0<d \mid n} d$.

$$
P_{g}=\sum_{t \in S_{N}} 2 k N_{t} \sum_{\mu_{t} \leq n<0} \sigma\left(-n / N_{t}\right) r_{t}(n)
$$

Proof. We begin by stating a lemma which was proved by Eholzer and Skoruppa in [4].

Lemma 2.2. Suppose that $f=\sum_{n=h}^{\infty} a(n) q^{n}$ is a meromorphic modular function in a neighborhood of $q=0$ and that $a(h)=1$. Then there are uniquely determined complex numbers $c(n)$ such that

$$
f=q^{h} \prod_{n=1}^{\infty}\left(1-q^{n}\right)^{c(n)},
$$

where the product converges in a small neighborhood of $q=0$. Moreover, the following identity is true

$$
\frac{\theta f}{f}=h-\sum_{n \geq 1} \sum_{d \mid n} c(d) d q^{n} .
$$

Let

$$
F(z)=\frac{\theta f(z)}{f(z)}-\frac{k}{12} E_{2}(z) .
$$

Here, $E_{2}(z)$ is the usual normalized Eisenstein series of weight 2 defined by

$$
E_{2}(z)=1-24 \sum_{n \geq 1} \sigma(n) q^{n} .
$$

The function $F(z)$ is a meromorphic modular form of weight 2 on $\Gamma_{0}(N)$. Its Fourier expansion at $t \in S_{N}$ is given by

$$
\begin{aligned}
\left.F(z)\right|_{2} \gamma_{t}^{(N)} & =\left.\left(\frac{\theta f(z)}{f(z)}\right)\right|_{2} \gamma_{t}^{(N)}-\left.\frac{k}{12} E_{2}(z)\right|_{2} \gamma_{t}^{(N)} \\
& =\frac{\theta\left(\left.f\right|_{k} \gamma_{t}^{(N)}\right)}{\left.f\right|_{k} \gamma_{t}^{(N)}}-\frac{k}{12} N_{t} E_{2}\left(N_{t} z\right) \\
& =h_{t}-\sum_{n \geq 1} \sum_{d \mid n} c_{t}(d) d q^{n}-\frac{k}{12} N_{t}+2 k N_{t} \sum_{n \geq 1} \sigma\left(n / N_{t}\right) q^{n} .
\end{aligned}
$$

Since $F(z) g(z) d z$ is a meromorphic 1-form on $X_{0}(N)$, we deduce that for $t \in S_{N}$,

$$
\begin{aligned}
2 \pi i \operatorname{Res}_{Q_{t}} F(z) g(z) d z= & \left(h_{t}-N_{t} \frac{k}{12}\right) r_{t}(0)+2 k N_{t} \sum_{\mu_{t} \leq n<0} \sigma\left(-n / N_{t}\right) r_{t}(n) \\
& -\sum_{\mu_{t} \leq n<0}\left(\sum_{d \mid-n} c_{t}(d) d\right) r_{t}(n)
\end{aligned}
$$

from (2.2). 
Next we compute $\operatorname{Res}_{Q_{\tau}} F(z) g(z) d z$ for $\tau \in \mathbb{H}$. For each $\tau \in \mathbb{H}$, from (2.1) we find that

$$
2 \pi i \operatorname{Res}_{Q_{\tau}} F(z) g(z) d z=2 \pi i \frac{1}{l_{\tau}} \operatorname{Res}_{\tau} \frac{\theta f(z)}{f(z)} g(z)=\nu_{\tau}^{(N)}(f) g(\tau)
$$

since $E_{2}(z)$ and $g(z)$ are holomorphic on $\mathbb{H}$.

The residue theorem implies that

$$
2 \pi i \sum_{Q_{\tau} \in X_{0}(N)} \operatorname{Res}_{Q_{\tau}} F(z) g(z) d z=0
$$

since $X_{0}(N)$ is a compact Riemann surface. This completes the proof of Theorem 2.1 .

REMARK 2.3. Suppose that $j(z)$ is the usual $j$-function

$$
j(z)=\frac{1}{q}+744+196884 q+\cdots
$$

and $j_{m}(z)=(j(z)-744) \mid T_{0}(m)$, where $T_{0}(m)$ is the normalized $m$ th weight zero Hecke operator. When $g(z)=j_{n}(z)$, Kohnen and Ahlgren point out that Theorem 2.1 can be extended to every finite index subgroup $G$ of $\Gamma$ in the following way. Let $S$ be the set of equivalence classes of cusps of $G$ and $h_{s}$ denote the period of $\left.f(z)\right|_{k} \gamma_{s}$ where $s=\gamma_{s} \infty$. Let $\left\{c_{s}(n)\right\}_{n=1}^{\infty}$ be the complex numbers for which

$$
\left.f\right|_{k} \gamma_{s}=q_{s}^{\mu_{s}} \prod_{n=1}^{\infty}\left(1-q_{s}^{n}\right)^{c_{s}(n)},
$$

where $q_{s}=q^{1 / h_{s}}$. Using the residue theorem with

$$
\begin{aligned}
j_{m}(z)\left(\frac{\theta\left(\left.\prod_{\gamma \in G \backslash \Gamma} f(z)\right|_{k} \gamma\right)}{\left.\prod_{\gamma \in G \backslash \Gamma} f(z)\right|_{k} \gamma}-[\Gamma: G] \frac{k}{12} E_{2}(z)\right) \\
=j_{m}(z)\left(\sum_{\gamma \in G \backslash \Gamma} \frac{\theta\left(\left.f(z)\right|_{k} \gamma\right)}{\left.f(z)\right|_{k} \gamma}-[\Gamma: G] \frac{k}{12} E_{2}(z)\right),
\end{aligned}
$$

we obtain

$$
\sum_{\tau \in G \backslash \mathbb{H}} \nu_{\tau}(f) j_{m}(\tau)+2 k \sigma(m)[\Gamma: G]=\sum_{s \in S} \sum_{d \mid m h_{s}} d c_{s}(d),
$$

where $\nu_{\tau}(f)=\left(1 / l_{\tau}\right) \operatorname{ord}_{\tau}(f)$ and $l_{\tau}$ is the order of the isotropy subgroup of $G$ at $\tau$.

We give an example of application of Theorem 2.1 for a meromorphic modular function $j_{m}^{(p)}=q^{-m}+\sum_{n=0}^{\infty} a_{n} q^{n}$ whose order of pole at every cusp except $\infty$ is bounded by a fixed constant for every positive integer $m$. 
EXAMPLE 2.4. Let

$$
\phi_{p}(z):=\left(\frac{\eta(z)}{\eta(p z)}\right)^{24 u /(p-1)} \quad \text { for a prime number } p
$$

where $u=(p-1) / \operatorname{gcd}(p-1,24)$ and $\eta(z)$ is defined by the product

$$
\eta(z)=q^{1 / 24} \prod_{n=1}^{\infty}\left(1-q^{n}\right)
$$

For each $m \geq 1$, let $j_{m}^{(p)}$ be a polynomial in $\phi_{p}(z)$ and $j(z)$ defined by

$$
j_{m}^{(p)}(z):=\phi_{p}(z)^{[m / u]} j(z)^{m-[m / u] u}+\sum_{n=0}^{m-1} b_{m}^{(p)}(n) \phi_{p}(z)^{[n / u]} j(z)^{n-[n / u] u}
$$

where the constants $b_{m}^{(p)}(n)$ are so chosen that $j_{m}^{(p)}$ has the $q$-expansion of the form

$$
j_{m}^{(p)}(z)=q^{-m}+\alpha_{m}^{(p)}(0)+\sum_{n=1}^{\infty} \alpha_{m}^{(p)}(n) q^{n} \quad \text { at } \infty
$$

and its $q$-expansion at 0 has no constant term. Let $\left(L_{i j}\right)_{(m+1) \times(m+1)}$ be the $(m+1) \times(m+1)$ matrix whose $i j$-entry is equal to the $(-j)$ th coefficient of $\phi_{p}(z)^{[(i-1) / u]} j(z)^{(i-1)-[(i-1) / u] u}$. Since $\left(L_{i j}\right)_{(m+1) \times(m+1)}$ is invertible, $b_{m}^{(p)}(i)$ is uniquely determined for $0 \leq i \leq m-1$. So, $j_{m}^{(p)}(z)$ is well defined. Let the $q$-expansion of $\left.j_{m}^{(p)}(z)\right|_{0}\left(\begin{array}{cc}0 & -1 \\ p & 0\end{array}\right)$ at $\infty$ be

$$
\left.j_{m}^{(p)}(z)\right|_{0}\left(\begin{array}{cc}
0 & -1 \\
p & 0
\end{array}\right)=\sum_{n=m_{0}}^{\infty} \beta_{m}^{(p)}(n) q^{n} \quad\left(\beta_{m}^{(p)}(0)=0\right) .
$$

First, note that $-p(u-1) \leq m_{0}$. Secondly, $j_{m}^{(p)}$ is holomorphic on $\mathbb{H}$ since $\phi_{p}(z)$ is holomorphic on $\mathbb{H}$ and $j_{m}^{(p)}$ is a polynomial in $j(z)$ and $\phi_{p}(z)$. So, if $f(z)$ is a meromorphic modular form of weight $k$ on $\Gamma_{0}(p)$, then for each $m \geq 1$ we have

$$
\begin{aligned}
\sum_{d \mid m} c_{\infty}(d) d+\sum_{m_{0} \leq n<0} & \left(\sum_{d \mid-n} c_{0}(d) d\right) \beta_{m}^{(p)}(n) \\
= & \sum_{\tau \in \mathcal{F}_{p}} \nu_{\tau}^{(p)}(f) j_{m}^{(p)}(\tau)+\left(h-\frac{k}{12}\right) \alpha_{m}^{(p)}(0)+2 k \sigma(m) \\
& +2 k p \sum_{m_{0} \leq n<0} \sigma(-n / p) \beta_{m}^{(p)}(n)
\end{aligned}
$$

The sequence $\left\{j_{m}^{(p)}\right\}$ is an example of an $(l, p)$-type sequence defined in the next section. 
3. Identities related to an $(l, p)$-type sequences. We begin by defining a class of sequences of modular functions.

Definition 3.1. Let $\left\{g_{m}^{(N)}\right\}$ be a sequence of modular functions on $\Gamma_{0}(N)$. We call $\left\{g_{m}^{(N)}\right\}$ an $(l, N)$-type sequence if:

(1) for every $m \geq 1$, the function $g_{m}^{(N)}$ is holomorphic on $\mathbb{H}$,

(2) $g_{m}^{(N)}=q^{-m}+\sum_{n=0}^{\infty} a_{m}(n) q^{n}$ at $\infty$,

(3) $-l=\inf \left\{m_{t} \mid t \in S_{N} \backslash\{\infty\}\right.$ and $\left.m \geq 1\right\} \gg-\infty$ if $N \neq 1, l=-1$ if $N=1$,

where $\left.g_{m}^{(N)}\right|_{0} \gamma_{t}^{(N)}=\sum_{n=m_{t}}^{\infty} a_{m}^{t}(n) q^{n}$ at $\infty$ and $a_{m}^{t}\left(m_{t}\right) \neq 0$.

The Riemann-Roch theorem implies that for each positive integer $N$ there exists an integer $I$ such that for any $l \geq I$ we can find an $(l, N)$-type sequence.

We define a function related to a sequence $\left\{g_{m}^{(N)}\right\}$ of modular functions on $\Gamma_{0}(N)$. Let

$$
\left[g_{m}^{(N)}\right]_{\tau}(z)=\sum_{n=1}^{\infty} g_{n}^{(N)}(\tau) q^{n} \quad \text { for } \tau \in \mathbb{H}
$$

and

$$
\left[g_{m}^{(N)}\right]_{t}(z)=\sum_{n=1}^{\infty} a_{n}^{t}(0) q^{n} \quad \text { for } t \in S_{N} \backslash\{\infty\},
$$

where $\left.g_{m}^{(N)}(z)\right|_{0} \gamma_{t}^{(N)}=\sum_{n=m_{t}}^{\infty} a_{m}^{t}(n) q^{n}$. For notational convenience we define

$$
\left[g_{m}^{(N)}\right]_{\infty}(z)=-1+\sum_{n=1}^{\infty} a_{n}(0) q^{n} .
$$

Though in general $\left[g_{m}^{(N)}\right]_{\tau}(z)$ is not a meromorphic modular form, we give some examples where $\left[g_{m}^{(N)}\right]_{\tau}(z)$ is a meromorphic modular form.

EXAmple $3.2($ see $[3])$. Let $j_{m}(z)=(j(z)-744) \mid T_{0}(m)$. A sequence $\left\{j_{m}(z)\right\}$ is a $(-1,1)$-type sequence, and we have

$$
\left[j_{m}\right]_{\tau}(z)=\frac{E_{4}^{2}(z) E_{6}(z)}{\Delta(z)} \cdot \frac{1}{j(z)-j(\tau)} \quad \text { for } \tau \in \mathbb{H},
$$

where $E_{k}(z)$ is the usual normalized Eisenstein series of weight $k$.

EXAmple 3.3. The sequence $\left\{j_{m}^{(2)}\right\}$ defined in Example 2.4 is a $(-1,2)$ type sequence. Let $\omega=(1+\sqrt{-3}) / 2$ and $f(z)=E_{4}(2 z)$. It is well known that $E_{4}(2 z)$ has only a zero at $\omega / 2$ in $\mathcal{F}_{2}$. A complex number $c_{t}(n)$ is defined 
from (2.3). Then we have

$$
\sum_{d \mid n} c_{\infty}(d) d=j_{n}^{(2)}(\omega / 2)-16 \sigma(n / 2)+16 \sigma(n),
$$

since $\left\{j_{m}^{(2)}\right\}$ is a $(-1,2)$-type sequence. Using this result and Ramanujan's identity (see [6]), we obtain

$$
\left[j_{m}^{(2)}\right]_{\omega}(z)=\frac{2}{3}\left(\frac{E_{6}(2 z)}{E_{4}(2 z)}-2 E_{2}(2 z)+E_{2}(z)\right) .
$$

In fact, when $p \in\{2,3,5,7,13\},\left[j_{m}^{(p)}\right]_{\tau}(z)$ is a meromorphic modular form for $\tau \in \mathbb{H}$. This can be deduced from Theorem 3.4 (or see [1]).

It can be shown that the $\theta$-operator plays an important role in the theory of modular forms and $p$-adic modular forms (see [7], [8]). Theorem 3.4 gives an expression of $\frac{\theta f}{f}$ through $\left[g_{m}^{(N)}\right]_{\tau}$ and $\left[g_{m}^{(N)}\right]_{\infty}$.

THEOREM 3.4. Suppose that $f(z)$ is a modular form of weight $k$ on $\Gamma_{0}(N)$, and that $\left\{g_{m}^{(N)}\right\}$ is an $(l, N)$-type sequence. For each cusp $t$ let $\left\{c_{t}(n)\right\}_{n=1}^{\infty}$ be the complex numbers for which

$$
\left.f\right|_{k} \gamma_{t}^{(N)}=\alpha_{t} q^{h_{t}} \prod_{n=1}^{\infty}\left(1-q^{n}\right)^{c_{t}(n)} \quad \text { for a complex number } \alpha_{t} .
$$

Then

$$
\begin{aligned}
& -\frac{\theta f(z)}{f(z)}+\frac{k}{12} E_{2}(z) \\
& =\sum_{\tau \in \mathcal{F}_{N}} \nu_{\tau}^{(p)}(f)\left[g_{m}^{(N)}\right]_{\tau}(z)+\sum_{t \in S_{N}}\left(h_{t}-N_{t} \frac{k}{12}\right)\left[g_{m}^{(N)}\right]_{t}(z) \\
& \quad-\sum_{m=1}^{\infty}\left(\sum_{t \in S_{N} \backslash\{\infty\}} \sum_{m_{t} \leq n<0} \sum_{d \mid-n} c_{t}(d) a_{m}^{t}(n) d-\left(P_{g_{m}^{(N)}}-2 k \sigma(m)\right)\right) q^{m}
\end{aligned}
$$

where $\left.g_{m}^{(N)}(z)\right|_{0} \gamma_{t}^{(N)}=\sum_{n=m_{t}}^{\infty} a_{m}^{t}(n) q^{n}$.

Proof. From Theorem 2.1 we have

$$
\begin{aligned}
\frac{\theta f(z)}{f(z)} & =h_{\infty}-\sum_{m=1}^{\infty} \sum_{d \mid m} c_{\infty}(d) d q^{m} \\
& =h_{\infty}-\sum_{m=1}^{\infty}\left(\sum_{\tau \in \mathcal{F}_{N}} \nu_{\tau}^{(p)}(f) g_{m}^{(N)}(\tau)+\sum_{t \in S_{N}}\left(h_{t}-N_{t} \frac{k}{12}\right) a_{m}^{t}(0)\right) q^{m}
\end{aligned}
$$




$$
\begin{aligned}
& -\sum_{m=1}^{\infty}\left(2 k \sigma(m)+\sum_{t \in S_{N} \backslash\{\infty\}} \sum_{m_{t} \leq n \leq 0}\left(2 k N_{t} \sigma\left(-n / N_{t}\right)-\sum_{d \mid-n} c_{t}(d) d\right) a_{m}^{t}(n)\right) q^{m} \\
= & -\sum_{\tau \in \mathcal{F}_{N}} \nu_{\tau}^{p}(f)\left[g_{m}^{(N)}\right]_{\tau}(z)-\sum_{t \in S_{N}}\left(h_{t}-N_{t} \frac{k}{12}\right)\left[g_{m}^{(N)}\right]_{t}(z)+\frac{k}{12} E_{2}(z) \\
& +\sum_{m=1}^{\infty}\left(\sum_{t \in S_{N} \backslash\{\infty\}} \sum_{m_{t} \leq n<0} \sum_{d \mid n} c_{t}(d) a_{m}^{t}(n) d-\left(P_{g_{m}^{(N)}}-2 k \sigma(m)\right)\right) q^{m} .
\end{aligned}
$$

So, the result follows.

When $\left\{g_{m}^{(p)}\right\}$ is an $(l, p)$-type sequence for a prime $p$, we can obtain an explicit formula for the sum of $\left[g_{m}^{(p)}\right]_{\tau}(z)$ and $\left[g_{m}^{(p)}\right]_{\infty}(z)$.

ThEOREM 3.5. Suppose that $\left\{g_{m}^{(p)}\right\}$ is an $(l, p)$-type sequence for a prime $p$ and an integer $l \geq-1$. Let

$$
u=\frac{p-1}{\operatorname{gcd}(p-1,24)}, \quad v=\max \left\{1,\left[\frac{l}{u}\right]+1\right\} .
$$

Then for $\tau_{0} \in \mathbb{H}$ we have

$$
\sum_{\tau \in W_{p}}\left[g_{m}^{(p)}\right]_{\tau}(z)-u v\left[g_{m}^{(p)}\right]_{\infty}(z)=\frac{u v}{p-1} \cdot \frac{\eta(z)^{24 u v /(p-1)}\left(p E_{2}(p z)-E_{2}(z)\right)}{\eta(z)^{24 u v /(p-1)}-\tau_{0} \eta(p z)^{24 u v /(p-1)}},
$$

where $W_{p}=\left\{\tau \in \mathcal{F}_{p} \mid \phi_{p}(z)^{v}=\tau_{0}, \phi_{p}(z):=(\eta(z) / \eta(p z))^{24 u /(p-1)}\right\}$.

Proof. To apply Theorem 3.4 we take $f(z)=\phi_{p}(\tau)^{v}-\tau_{0}$ for $\tau_{0} \in \mathbb{H}$. Suppose that $g_{m}^{(p)}(z)$ has the $q$-expansion

$$
\begin{aligned}
& g_{m}^{(p)}=q^{-m}+\sum_{n=0}^{\infty} a_{m}^{\infty}(n) q^{n} \quad \text { at } \infty \\
& \left.g_{m}^{(p)}\right|_{0} \gamma_{0}^{(p)}=\sum_{n=m_{0}}^{\infty} a_{m}^{0}(n) q^{n} \quad \text { at } \infty .
\end{aligned}
$$

For each cusp $t$ let $\left\{c_{t}(n)\right\}_{n=1}^{\infty}$ be the complex numbers for which

$$
\left.f\right|_{k} \gamma_{t}^{(N)}=\alpha_{t} q^{h_{t}} \prod_{n=1}^{\infty}\left(1-q^{n}\right)^{c_{t}(n)} \quad \text { for a complex number } \alpha_{t} .
$$

Here, $h_{\infty}=-u v$ and $h_{0}=0$. Note that the $n$th Fourier coefficient of $\left.\phi_{p}(\tau)^{v}\right|_{0} \gamma_{0}^{(p)}$ is zero for every $n \leq l$. This implies that $\theta\left(\left.f\right|_{0} \gamma_{0}\right)$ has a zero of order at least $l+1$ at $\infty$. So, the function $\left.\frac{\theta f}{f}\right|_{2} \gamma_{0}$ has the $q$-expansion

$$
\left.\frac{\theta f}{f}\right|_{2} \gamma_{0}=\frac{\theta\left(\left.f\right|_{0} \gamma_{0}\right)}{\left.f\right|_{0} \gamma_{0}}=\sum_{d \mid(l+1)} c_{0}(d) d q^{l+1}+O\left(q^{l+2}\right) .
$$


Since $\left\{g_{m}^{(p)}(z)\right\}$ is an $(l, p)$-type sequence, for every $m \geq 1$ we obtain

$$
\sum_{m_{0} \leq n<0}\left(\sum_{d \mid-n} c_{0}(d) d\right) a_{m}^{0}(n)=0 .
$$

Moreover, for every $m \geq 1$, we have $P_{g_{m}^{(p)}}=0$ by noting that the weight of $f$ is zero. Therefore, from Theorem 3.4 we obtain

$$
\begin{aligned}
\sum_{\tau \in W_{p}}\left[g_{m}^{(p)}\right]_{\tau}(z)-u v\left[g_{m}^{(p)}\right]_{\infty}(z) & =-\frac{\theta f}{f} \\
& =\frac{24 u v}{p-1} \cdot \frac{\phi_{p}(z)^{v}\left(\frac{p}{2 \pi i} \frac{\eta^{\prime}(p z)}{\eta(p z)}-\frac{1}{2 \pi i} \frac{\eta^{\prime}(z)}{\eta(z)}\right)}{\phi_{p}(z)^{v}-\tau_{0}} \\
& =\frac{u v}{p-1} \cdot \frac{\eta(z)^{24 u v /(p-1)}\left(p E_{2}(p z)-E_{2}(z)\right)}{\eta(z)^{24 u v /(p-1)}-\tau_{0} \eta(p z)^{24 u v /(p-1)}}
\end{aligned}
$$

since

$$
\frac{\eta^{\prime}(z)}{\eta(z)}=\frac{2 \pi i}{24} E_{2}(z)
$$

Remark 3.6. Suppose that $p \in\{2,3,5,7,13\}$. Take $g_{m}^{(p)}=\left\{j_{m}^{(p)}\right\}$ defined in Example 2.4. The sequence $\left\{j_{m}^{(p)}\right\}$ is a $(-1, p)$-type sequence, and

$$
\left[j_{m}^{(p)}\right]_{\infty}(z)=\frac{-1}{p-1}\left(E_{2}(z)-p E_{2}(p z)\right) .
$$

Take $\tau_{0}=\phi_{p}(\tau)$ for a fixed $\tau \in \mathbb{H}$. Since $\phi_{p}$ is bijective from $\mathcal{F}_{p}$ to $\mathbb{C} \backslash\{0\}$, we have $W_{p}=\{\tau\}$. Then we obtain

$$
\left[j_{m}^{(p)}\right]_{\tau}(z)=\frac{p E_{2}(p z)-E_{2}(z)}{p-1}\left(\frac{\eta(z)^{24 /(p-1)}}{\eta(z)^{24 /(p-1)}-\phi_{p}(\tau) \eta(p z)^{24 /(p-1)}}-1\right)
$$

from Theorem 3.5. So, in this case we recover the result given in [1]. Furthermore, Theorem 1 in [1] turns out to be a special case of Theorem 3.5.

4. Values of modular functions at certain points. In this section, we introduce some congruence properties of $j_{m}^{(p)}(\tau)$ at the zero divisor of $E_{k}$. The von Staudt-Clausen Theorem (see p. 153 in [5]) gives the congruence properties of $E_{k}(z)$ : for each $k \geq 4$,

$$
E_{k} \equiv 1\left(\bmod 4 \prod_{\substack{(e-1) \mid k \\ 5 \leq e \text { prime }}} e\right)
$$

From this identity, when $p \in\{2,3,5,7,13\}$, we obtain congruence properties of the values of $j_{m}^{(p)}$ defined in Example 2.4 at a certain point. 
Corollary 4.1. Suppose that $p \in\{2,3,5,7,13\}$. Let $f$ be a normalized modular form of weight $\delta$ such that

$$
f=\sum_{i=1}^{h} c_{i} \prod_{j=1}^{t_{i}} E_{k_{i j}}\left(L_{i j} z\right) \quad \text { for } c_{i} \in \mathbb{Z} \text { and } L_{i j} \mid p,
$$

where $k_{i j} \geq 4$ is a positive even integer. Let $k$ be the greatest common divisor of the $k_{i j}$. If $\operatorname{gcd}(e, p-1)=1$ for every e such that $e \geq 5$ and $(e-1) \mid k$, then

$$
\begin{aligned}
\sum_{\tau \in \mathcal{F}_{p}} \nu_{\tau}^{(p)}(f) j_{m}^{(p)}(\tau) & \\
& \equiv \frac{2 \delta p}{p-1} \sigma(m(p-1) / p)-\frac{2 \delta p}{p-1} \sigma(m(p-1))\left(\bmod \prod_{\substack{(e-1) \mid k \\
5 \leq e \text { prime }}} e\right) .
\end{aligned}
$$

Proof. Using the von Staudt-Clausen Theorem, for even $r \geq 4$ we have

$$
E_{r} \equiv 1\left(\bmod 4 \prod_{\substack{(e-1) \mid r \\ 5 \leq e \text { prime }}} e\right) \text {. }
$$

This identity implies

$$
\frac{\theta f}{f} \equiv 0\left(\bmod 4 \prod_{\substack{(e-1) \mid k \\ 5 \leq e \text { prime }}} e\right) .
$$

From Theorem 2.1 we obtain

$$
\begin{aligned}
& \sum_{\tau \in \mathcal{F}_{p}} \nu_{\tau}^{(p)}(f) j_{m}^{(p)}(\tau) \\
& \quad \equiv \frac{2 \delta p}{p-1} \sigma(m(p-1) / p)-\frac{2 \delta p}{p-1} \sigma(m(p-1))\left(\bmod \prod_{\substack{(e-1) \mid k \\
5 \leq e \text { prime }}} e\right) .
\end{aligned}
$$

Corollary 4.2. Suppose that $p \in\{2,3,5,7,13\}$. Let $j_{m}^{(p)}$ be a modular function defined in Example 2.4 and let $\omega$ denote $(1+\sqrt{-3}) / 2$.

(I) Let $T:=\left\{\gamma \omega \in \mathcal{F}_{p} \mid \gamma \in \mathrm{SL}_{2}(\mathbb{Z})\right\}$ and $M$ be a positive integer not divisible by a prime $\mathcal{P} \equiv 1(\bmod 3)$. If $\operatorname{gcd}(p-1, M)=1$, then for some positive real number $\xi(M)$ we have

$$
\begin{aligned}
\sharp\{1 \leq m & \leq X \mid 3\left(\sum_{\tau \in T} \frac{1}{l_{\tau}} j_{m}^{(p)}(\tau)\right) \\
& \left.\equiv \frac{24}{p-1}(p \sigma(m / p)-\sigma(m))(\bmod M)\right\}=\mathcal{O}\left(\frac{X}{(\log X)^{\xi(M)}}\right) .
\end{aligned}
$$


(II) Let $T:=\left\{\gamma i \in \mathcal{F}_{p} \mid \gamma \in \mathrm{SL}_{2}(\mathbb{Z})\right\}$ and $M$ be a positive integer not divisible by a prime $\mathcal{P} \equiv 1(\bmod 4)$. If $\operatorname{gcd}(p-1, M)=1$, then for some positive real number $\xi(M)$ we obtain

$$
\begin{aligned}
\sharp\{1 \leq m & \leq X \mid 2\left(\sum_{\tau \in T} \frac{1}{l_{\tau}} j_{m}^{(p)}(\tau)\right) \\
& \left.\equiv \frac{24}{p-1}(p \sigma(m / p)-\sigma(m))(\bmod M)\right\}=\mathcal{O}\left(\frac{X}{(\log X)^{\xi(M)}}\right) .
\end{aligned}
$$

Proof. Let $j_{m}(z)=(j(z)-744) \mid T_{0}(m)$. Applying Theorem 2.1 to $j_{m}^{(p)}$, we obtain

$$
\begin{aligned}
& 3\left(\sum_{\tau \in \mathcal{F}_{N}} \nu_{\tau}^{(p)}\left(E_{4}\right) j_{m}^{(p)}(\tau)\right)-\frac{24}{p-1}(p \sigma(m / p)-\sigma(m))=j_{m}(\omega), \\
& 2\left(\sum_{\tau \in \mathcal{F}_{p}} \nu_{\tau}^{(p)}\left(E_{6}\right) j_{m}^{(p)}(\tau)\right)-\frac{24}{p-1}(p \sigma(m / p)-\sigma(m))=j_{m}(i) .
\end{aligned}
$$

Theorem 6 in [3] implies that for each $\tau \in\{i, \omega\}$ there is a positive number $\xi(M)$ for which

$$
\sharp\left\{1 \leq m \leq X \mid j_{m}(\tau) \equiv 0(\bmod M)\right\}=\mathcal{O}\left(\frac{X}{(\log X)^{\xi(M)}}\right),
$$

where $M$ is as in (I) if $\tau=\omega$, and as in (II) if $\tau=i$. Therefore, the corollary can be proved by the method of Theorem 6 in [3]. So, we omit the details.

Acknowledgements. I would like to thank Professors W. Kohnen and S. Ahlgren for helpful comments and allowing me to mention their results. I am grateful to Professor Y. Choie for the various instructive discussions and introducing me to this object. I also appreciate the referee for a careful reading of the manuscript and for helpful comments.

\section{References}

[1] S. Ahlgren, The theta-operator and the divisors of modular forms on genus zero subgroups, Math. Res. Lett. 10 (2003), 787-798.

[2] R. E. Borcherds, Automorphic forms on $\mathcal{O}_{s+2,2}(\mathbb{R})$ and infinite products, Invent. Math. 120 (1995), 161-213.

[3] J. Bruinier, W. Kohnen and K. Ono, The arithmetic of the values of modular functions and the divisors of modular forms, Compos. Math. 140 (2004), 552-566.

[4] W. Eholzer and N.-P. Skoruppa, Product expansions of conformal characters, Phys. Lett. B 388 (1996), 82-89.

[5] S. Lang, Introduction to Modular Forms, Grundlehren Math. Wiss. 222, Springer, 1976.

[6] S. Ramanujan, On certain arithmetical functions, Trans. Cambridge Philos. Soc. 22 (1916), 159-184. 
[7] J.-P. Serre, Formes modulaires et fonctions zêta p-adiques, in: Lecture Notes in Math. 350, Springer, 1973, 191-268.

[8] H. P. F. Swinnerton-Dyer, On l-adic representations and congruences for coefficients of modular forms, ibid., 1-55.

[9] D. Zagier, Traces of singular moduli, in: Motives, Polylogarithms and Hodge Theory, Part I, Int. Press Lect. Ser. 3, I, Int. Press, Somerville, MA, 2002, 211-244.

Department of Mathematics

Pohang University of Science and Technology

Pohang, 790-784, Korea

E-mail: choija@postech.ac.kr

Received on 18.6.2004

and in revised form on 9.9.2005 